\title{
1 A LAMP-based microfluidic chip for rapid detection of pathogen in Cryptococcal
}

\section{2 meningitis}

3

4 Yueru Tian ${ }^{1 \dagger}$, Tong Zhang ${ }^{2 \dagger}$, Jian Guo ${ }^{3}$, Huijun $\mathrm{Lu}^{2}$, Yuhan $\mathrm{Yao}^{2}, \mathrm{Xi} \mathrm{Chen}^{2}$, Xinlian $5 \quad$ Zhang $^{2}$, Guodong Sui $^{2} *$, Ming Guan ${ }^{1} *$

6 1. Department of Laboratory Medicine, Huashan Hospital, Shanghai Medical College,

$7 \quad$ Fudan University, Shanghai, China. Email:vicky8025@126.com.

8 2. Department of Environmental Science \& Engineering, Fudan University, Shanghai, China. Department of Medical Microbiology \& Parasitology, Institute of Biomedical Science, Basic Medical College of Fudan University, Shanghai, China. Email: shmzhyzt@163.com, 19110740007@fudan.edu.cn, 16110740002@fudan.edu.cn, 18210740002@fudan.edu.cn,17110740037@fudan.edu.cn.

3. Department of Laboratory Medicine, Shanghai East Hospital, Tongji University School of Medicine, Shanghai, China. Email: guojian1110@126.com.

${ }^{\dagger}$ These authors contributed equally to this work.

*Correspondence should be addressed to Guodong Sui (gsui@fudan.edu.cn) or Ming Guan (guanming88@126.com)

Abstract: Cryptococcal meningitis (CM) is a global threat with significant attributable morbidity and mortality. Information on integrated detection for CM diagnosis is still limited. This is mainly due to the presence of a large polysaccharide capsule and the tough cell wall of Cryptococcus, which makes it difficult to extract nucleic acids on the chip. In this study, we developed a LAMP-based microfluidic chip for rapid detection of 
capture and simplify the enrichment process, and combined lyticase digestion and thermal alkaline lysisto optimize the nucleic acid extraction of Cryptococcus on the chip, and selected a portable UVA flashlight to shine the LAMP products to obtain the visual detection results which could be observed by the naked eye. This microfluidic chip,

30 integrating sample Cryptococcus enrichment, nucleic acid extraction and LAMP

31 detection unit, streamlined the operation process and reduced the exposure risk of directly handling cryptococcal samples. It did not require any additional instruments and demonstrated a rapid, reliable, as well as high-efficiency approach. It truly realized the "sample-to-answer" application and could be easily used for clinical cryptococcal prediagnosis.

KEYWORDS: Microfluidic chip, LAMP, Cryptococcal meningitis

\section{Introduction}

Cryptococcus is an opportunistic pathogenic fungus that resides in diverse ecological 40 niches, especially abundant in eucalyptus, avian excreta and amoeba. Among the 70

41 species identified, $C$. neoformans and $C$. gatti are the major causative agents of human cryptococcosis[1]. Found widely in soil, decaying wood, and bird droppings, pneumonia in immunocompromised patients, but in immunocompetent hosts the fungal cells are either cleared by the immune system or establish an asymptomatic latent

46 infection. Once the infected subject becomes immunosuppressed, the latent infection can

47 disseminate to other tissues, most notably the central nervous system (CNS), causing 48 life-threatening subacute meningitis[1].

49 Cryptococcal meningitis (CM) is the most common cause of HIV-related meningitis, 
51 CI 119 400-234 300) deaths per year[2]. Globally, CM was responsible for 15\% of

52 HIV-related deaths[2]. In recent years, an increasing number of CM cases have been

53 reported in non-HIV infected individuals, mainly including patients with natural and

54 iatrogenic immunosuppression, such as patients with organ or stem-cell transplantation,

55 malignant tumors, autoimmune diseases, glucocorticoid or immunosuppressive therapies

56 (including chemo-, radio-, or immunotherapy for cancer), or even "immunocompetent

57 individuals" carrying underlying immune deficiencies, chronic liver, kidney or lung

disease and diabetes[3]. In-hospital mortality of non-HIV-related CM has now reached

approximately $25 \%$ [4,5]. However, there are still about $65-70 \%$ of non-HIV CM

60 patients without any predisposing factors, particularly in China[6-8], Korea[9], Japan[10]

61 and India[11]. Moreover, $C$. gattii has been considered as the culprit causing CM in immunocompetent hosts, highlighted in Australia, Canada, and the U.S. Pacific

63 Northwest[12]. Worldwide, the mortality rate of patients with C. gattii infections ranges from $13 \%$ to $33 \%[12]$.

Currently, the diagnosis of $\mathrm{CM}$ still poses some difficulties. One is attributed to atypical symptoms (fever, headache, nausea, vomiting), which are easily confused with upper respiratory tract infection, tuberculous meningitis, viral meningitis or neurological diseases. Another is that conventional CM diagnostic techniques have some limitations. Both of these challenges lead to delayed diagnosis and higher morbidity and mortality. To date, diagnosis of CM has mainly relied on cerebrospinal fluid (CSF) India ink 71 microscopic staining, culture, cryptococcal anhtigen ( $\mathrm{CrAg})$ testing or histopathological examination. Although india ink microscopic staining has been a quick method, unfortunately sensitivity is low (86\% in expert hands)[13]. The cultivation technique is

74 time-consuming and usually takes 3-7 days to report, and once antibiotic treatment is established, false negative results are prone to occur (the sensitivity is approximately 
$7678.4 \%$ )[13]. CrAg detection has a sensitivity and specificity of 99\% in CSF, but latex agglutination (LA) is more expensive, more labor-intensive, and requires cold-chain shipping/storage[14]. Patients with rheumatoid factor positive, tuberculous meningitis or systemic lupus erythematosus may have a false positive reaction. The LA antigen titer of patients infected with Trichosporon can reach 1:1000[15]. Moreover, hemolytic samples and "hook effect" caused by high concentrations of CrAg can lead to false negative results in lateral flowassay (LFA)[16]. Histopathological examinations are complicated in preparation and staining processes, and microscopic examinations require experienced staff. Therefore, it is an urgent need to develop a rapid and sensitive diagnostic technique to complement the deficiencies of existing methods.

With the characteristics of rapid and high sensitivity, various polymerase chain reaction (PCR) technologies, such as nested PCR[17], real-time PCR[18,19], and singleplex PCR[20], have been applied to CM diagnosis. However, the extraction process of cryptococcal nucleic acid is labor intensive and cumbersome. Direct operation of samples containing Cryptococcus also increases the risk of exposures. Therefore, the development of integrated molecular amplification detection of cryptococcal samples is of great significance. Among the existing detection technology, nested PCR shows high specificity, but it must be carried out in two steps. Real-time PCR requires the establishment of a standard curve, internal reference and precise temperature control. All of these limit the possibility of developing integrated detection. Loop-mediated isothermal amplification (LAMP) is a good way to avoid these limitations. It is characterized by simple operation, isothermal amplification, short time required, simple equipment, and has shown promising potential in integrated detection applications.

$$
\text { Microfluidic chips integrated with LAMP assay for detecting bacterial meningitis, such }
$$
as meningitis caused by Neisseria meningitides[21,22], Streptococcus pneumoniae[21] 
103 dedicated to any link in sample pretreatment (pathogen enrichment) or amplification

104 method (isothermal amplification) or signal detection (colloidal gold enhanced

105 signal)[23-26], but few solutions are available for CM detection, mainly due to the tough

106 cell wall of Cryptococcus and the presence of large polysaccharide capsule (accounting

107 for approximately $70 \%$ of the whole cellular volume) in its outer layer, which makes it

108 difficult to achieve successful nucleic acid extraction in the chip. Various methods

109 proposed to extract and purify DNA from Cryptococcus included bead rupture method,

110 enzymatic cell wall lysis method and chemical reagents lysing method, yet the first

111 method is difficult to implement in a functional chip due to its mechanical properties. To

112 solve this issue, we combined the use of lyticase digestion, and thermal alkaline lysis in

113 the microfluidic chip to achieve effective nucleic acid extraction. In addition, we used a

114 filter membrane to capture the target and a portable UVA flashlight to read the amplified

115 signal, thus simplifying the entire operation process.

116 Here we developed a LAMP-based microfluidic chip to combine rapid filter

117 membrane-based sample concentration, nucleic acid extraction, target DNA amplification,

118 and portable UVA flashlight signal reading of pathogens from CSF samples, which

119 demonstrated a rapid, high-efficiency approach, and enabled "sample-to-answer"

120 detection from real CSF samples.

122 Materials and Methods

\section{CSF samples}

124 Between March 2019 and November 2019, 83 clinical CSF samples that were LFA 125 positive (CrAg titers ranging 1:1-1:20,480) were collected from CM patients of Shanghai 
126 Huashan Hospital in China. A total of 40 clinical positive-cultured CSF samples collected

127 from patients with other CNS infections (diagnosed by culture or pathology) (Table S1).

128 Sterile human CSF was collected from a non-infected patient who required CSF drainage

129 due to high CSF pressure, with his consent in Shanghai Huashan Hospital. With ethical

130 approval (accession number: KY2019 HIRB-002), all patients involved signed informed

131 consent forms, understanding and agreeing to use their clinical samples in this study.

\section{Isolates}

134 Eleven standard strains containing different serotypes, genotypes and AFLP types were 135 donated by Professor Min Chen (Table S2). Fifty strains used for specificity verification

136 were collected from Shanghai Huashan Hospital and confirmed by MALDI-TOF mass 137 spectrometry (Bruker Daltonics, Bremen, Germany) and nucleic acid sequence. (Table 138 S3).

\section{Standard Nucleic acid extraction}

141 Genomic DNA from cultured microorganisms was extracted using the Yeast DNA Kit

142 (Omega Bio-Tek, Norcross, GA). Genomic DNA from the clinical samples was extracted

143 using the QIAamp DNA Mini Kit (QIAGEN, Hilden, Germany) with some modifications:

144 an initial incubation with lyticase $(10 \mathrm{U})$ at $30{ }^{\circ} \mathrm{C}$ for $45 \mathrm{~min}$, followed by $\mathrm{AL}$ buffer 145 incubation at $90{ }^{\circ} \mathrm{C}$ for $10 \mathrm{~min}$, was included. The concentration of DNA was evaluated 146 using Qubit 3.0 fluorometer (Invitrogen, ThermoFisher Scientific, Malaysia).

148 LAMP assay performance verification

149 LAMP primers were designed using Primer Explorer V4 software

150 (http://primerexplorer.jp/e/) based on the Cryptococcus capsular-associated protein 10 $6 / 30$ 
gene (CAP10) (Table 1). The assay was run on an ABI Prism 7500 real-time PCR system

152 (Applied Biosystems, USA). The reaction was carried out in a $25 \mu 1$ reaction mixture containing $13 \mu \mathrm{l} 1 \times$ ThermoPol Buffer $(20 \mathrm{mM}$ Tris-HCl, $50 \mathrm{mM} \mathrm{KCl}, 10 \mathrm{mM}$ (NH4) $)_{2} \mathrm{SO}_{4}, 2 \mathrm{mM} \mathrm{MgSO}$, $0.1 \%$ Tween 20, $6 \mathrm{mM} \mathrm{MgSO} 4,1.4 \mathrm{mM}$ dNTP mixture, 5×SYBR Green), $1 \mu$ target-specific primer mixture $(1.6 \mu \mathrm{M}$ FIP/BIP, and $0.2 \mu \mathrm{M}$ F3/B3), $1 \mu 18 \mathrm{U}$ of Bst DNA polymerase and $10 \mu 1$ of DNA. The mixture was incubated at $64.8{ }^{\circ} \mathrm{C}$ for $5 \mathrm{~s}, 65{ }^{\circ} \mathrm{C}$ for $55 \mathrm{~s}$ (collecting the fluorescent) for 45 minutes. The sterile CSF and ultra-purified water were used as negative controls. As a positive control, $1 \mathrm{ng} / \mu \mathrm{l}$ purified C. neoformans DNA was used.

160 Evaluation of the accuracy of the LAMP assay: Eleven standard strains containing 161 different serotypes, genotypes and AFLP types were adjusted to 0.5 McFarland standard suspensions and DNA was extracted for LAMP accuracy verification.

163 Evaluation of the specificity of the LAMP assay: DNA extracted from forty clinical 164 positive-cultured CSF samples collected from patients with other CNS infections

165 (including Enterobacteriaceae, Acinetobacter, Staphylococcus, Enterococcus, Listeria sp,

166 Candida, Nocardia, Mycobacterium, Aspergillus, Cysticercus and complex infection)

167 (Table S1), one sterile human CSF sample (negative control), one ultra-filtered water 168 (negative control), and fifty pure cultures of microorganisms (including different species 169 and closely related species) (Table S3), was tested for LAMP specificity.

170 Evaluation of the detection limits of the LAMP assay: Continuously dilute (1:10) the $C$. 171 neoformans yeast suspensions and its DNA into sterile CSF ranging from $10^{8} \mathrm{CFU} / \mathrm{ml}$ to $1721 \mathrm{CFU} / \mathrm{ml}$ and $1 \mathrm{ng} / \mu \mathrm{l}$ to $1 \mathrm{fg} / \mu \mathrm{l}$, then extract the DNA and use it for sensitivity 173 verification.

174 Evaluation of the precision of the LAMP assay: The precision assay was divided into 175 intra-batch and inter-batch trials. The intra-batch trial selects $201 \mathrm{pg} / \mu 1$ weak positive 
176 samples and 20 ultra-filtered water samples for testing together. The inter-batch trial

177 selects one $1 \mathrm{pg} / \mu 1$ weakly positive sample and one ultra-filtered water sample and tests

178 once a day for 20 days.

179 Analysis of clinical samples: DNA extracted from 83 LFA-positive CSF samples was

180 used as a template for LAMP assay.

181

\section{Chip fabrication}

183 All the microchannels and the reaction holes were fabricated using soft lithography. The

184 PDMS (Momentive, NY, USA) prepolymer and curing agent were mixed at a ratio of 5:1

185 (w:w) and 7:1 (w:w) to prepare different layers. The mixture was casted onto a silicon

186 wafer having different patterns of SU-8 2100 (MicroChem, MA, USA) and AZ-50 (AZ

187 Electronic Materials, Merck, USA) and cured at $80^{\circ} \mathrm{C}$, afterwards the cured PDMS layer

188 was peeled off from the wafer. Holes in reaction layer, mixed layers and filtration layers

189 were punched with $2 \mathrm{~mm}$ and $5 \mathrm{~mm}$ perforators respectively. The $1 \mu \mathrm{m}$ pore diameter

190 sized polycarbonate membranes (General Electric, WI, USA) were sealed between the

191 mixed layers and cured at $80{ }^{\circ} \mathrm{C}$ for $6 \mathrm{~h}$. A ratio of 10:1 PDMS prepolymer/curing agent

192 was injected into the filling channel through the filling inlet and was cured at $120^{\circ} \mathrm{C}$ for

1935 min to solidify the filtration layers sandwich. Then, the reaction layer, collection layers,

194 mixed layers and filtration layers were aligned and heated sealing together at $80{ }^{\circ} \mathrm{C}$

195 overnight.

196

197 On-chip nucleic acid extraction

198 In order to obtain free nucleic acid in microfluidic chip, we combined lyticase, Qiagen

199 DNA Kit AL buffer (QIAGEN, Hilden, Germany), Novagen Bugbuster Master Mix 
200 (Novagen, Merck, USA) and three additives (NaOH, SDS, urea) to select the best

201 extraction strategy. Besides, temperature was another influencing factor.

202

\section{On-chip LAMP amplification and signal detection}

204 After nucleic acid extraction, the LAMP reaction solution and nucleic acid were injected

205 into the reaction wells. Polyester (PET) film (General Electric, WI, USA) sealed the top 206 layer, and the integrated chip was incubated in an oven at $65{ }^{\circ} \mathrm{C}$ for 45 minutes. After

207 on-chip LAMP amplifications, a portable UV flashlight was applied to shine LAMP 208 products to obtain the visual detection results. The generated fluorescence could be 209 observed by the naked eye.

\section{Results}

\section{LAMP assay performance verification}

213 The accuracy of the LAMP assay: Analysis of 11 different serotypes, genotypes and

214 AFLP types of $C$. neoformans/gattii standard strains showed that accuracy was $100 \%$ 215 (Table S2).

216 The specificity of the LAMP assay: The results showed that the specificity of the assay 217 is $100 \%$, as both the 40 clinical samples from patients with other CNS infections and 2 218 negative controls (0 positive/42 samples) (Table $\mathrm{S} 1$ ) gave negative results, and no 219 cross-reaction was observed in 50 other pure cultured microorganisms (0/50) (Table S3).

220 The detection limits of the LAMP assay: The optimized LAMP assay conditions in our 221 system allowed detection of $100 \mathrm{fg} / \mu \mathrm{l}$ of Cryptococcus DNA (Figure 1A) and 100 222 CFU/ml of Cryptococcus yeast suspension (Figure 1B).

223 The precision of the LAMP assay: In intra-batch and inter-batch precision tests, 20 weakly positive samples $(1 \mathrm{pg} / \mu \mathrm{l})$ were all positive, 20 negative samples were all negative, 
and the agreement rates were $100 \%$. The average positive time of the respective intra-batch and inter-batch assay was $26.82 / 27.33 \mathrm{~min}$, SD value was $1.70 / 3.02$, and CV value was $6.33 \% / 11.06 \%$.

Analysis of clinical samples: The total positive rate of $83 \mathrm{CSF}$ samples detected by LAMP PCR was $91.6 \%$ (76/83). The positive rate of LAMP assay with LFA titer in the range of 1:80-1:2,040 was $100 \%$, while those of lower titers (1:1-1:40) was $33.3-90.0 \%$ (Table 2).

\section{Microfluidic chip pathogen enrichment}

234 This core area was composed of 4 duplicate filtration membrane structures (Figure2).

235 PDMS/curing reagent mixtures were injected through the filling inlet and the protruding

237 injection more easily. The CSF sample was injected into the inlet and evenly distributed 238 to four enrichment zones. After washing with deionized water, pathogens were 239 effectively enriched and purified (Figure 3).

\section{Optimization of the on-chip nucleic acid extraction strategy}

242 Three different additives (NaOH, SDS and urea) were tested using standard Qiagen AL

243 buffer, and it was found that urea and SDS inhibit the amplification if removed unclearly.

244 Compared with other two additives, $0.5 \mathrm{M} \mathrm{NaOH}$ improved the extraction efficiency

245 (Figure 4A). We also compared the mild Bugbuster buffer (which was widely used to 246 lyse bacteria and had little influence on amplification) with Qiagen AL buffer using

$247 \mathrm{NaOH}$ additive and found that the lysis capacity of Bugbuster buffer was not enough for

248 Cryptococcus (data not shown). Moreover, considering the tolerance of the chip 249 temperature, we selected $25{ }^{\circ} \mathrm{C}, 45{ }^{\circ} \mathrm{C}, 65{ }^{\circ} \mathrm{C}$ and $85{ }^{\circ} \mathrm{C}$ as test points under the 
250 aforementioned conditions, and incubated the lysing mixture for 5 minutes. The

251 amplification results showed that the lysis efficiency could be improved under incubation

252 at $65{ }^{\circ} \mathrm{C}$ or $85{ }^{\circ} \mathrm{C}$ (Figure $4 \mathrm{~B}$ ). To simplify the process, we chose $65^{\circ} \mathrm{C}$ as the lysis

253 temperature. Then, we explored the experimental effects of different concentrations of

254 lyticase to optimize the best working concentration. The results showed that $40 \mathrm{U} / \mathrm{ml}$

255 lyticase digested Cryptococcus at $30{ }^{\circ} \mathrm{C}$ for 30 minutes in the $1 \mathrm{M}$ sorbitol buffer environment, and the amplification effect was the best (Figure 4C).

\section{Performance of the chip}

259 The integrated chip was composed of functional layers (the reaction layer, collection

260 layers, mixed layers and filtration layers) and other different layers. The illustration and

261 the entire operation process were shown in Figure 2, Figure 5 and supplementary material

262 video1. The sample was loaded into the filter well. After Cryptococcus enrichment, $300 \mu 1$

263 sterile deionized water was injected to wash the membrane to remove the matrix. The

264 liquid was evacuated with air, and approximately $60 \mu \mathrm{l}$ of $1 \mathrm{M}$ sorbitol buffer containing

$26540 \mathrm{U} / \mathrm{ml}$ lyticase was injected through the inlet, and withdrawn from the outlet. After 5

266 minutes of repeated suction operation, the filter membrane structure rich in pathogens

267 was evenly immersed in the buffer environment, and incubated at $30{ }^{\circ} \mathrm{C}$ for 30 minutes.

268 Subsequently, $60 \mu \mathrm{l}$ of AL buffer containing $0.5 \mathrm{M} \mathrm{NaOH}$ was injected. Repeated the 269 above suction operation, and after the lysis buffer was in full contact with the

270 Cryptococcus, incubated the chip at $65{ }^{\circ} \mathrm{C}$ for 5 minutes to release the nucleic acid.

271 Then, $100 \mu \mathrm{l}$ of $100 \mathrm{mM}$ PH 8.0 Tris- $\mathrm{HCl}$ buffer was injected through the outlet, and the

272 lysis buffer was neutralized by completely mixing from the top layer to the bottom layer

273 to release free nucleic acids. The LAMP reaction solution and DNA were injected into

274 the reaction well for isothermal amplification for 45 minutes. After 45 minutes of 
amplification, the detection results could be successfully read by the naked eye (Figure

276 6).

\section{Discussion}

278 Cryptococcal meningitis is a global threat with significant attributable mortality.

279 Worldwide, CM is typically associated with HIV infection[2], and it is increasingly

280 recognized in patients without HIV[6-11]. To date, information about integrated detection

281 for CM diagnosis is still limited. In this study, we developed a LAMP-based microfluidic

282 chip for the diagnosis of CM.

283 In order to ensure the accuracy and reliability of the LAMP system, we conducted

284 LAMP assay performance verification. We collected 11 different serotypes, genotypes and AFLP types of $C$. neoformans/gattii standard strains for accuracy verification, which was $100 \%$ and showed its high accuracy. The agreement rates of intra-batch and

287 inter-batch precision tests were $100 \%$. The average positive time of the intra-batch and 288 intra-batch assay was respectively $26.82 / 27.33 \mathrm{~min}$, SD value was $1.70 / 3.02$, and CV 289 value was $6.33 \% / 11.06 \%$, which showed relatively stable reproducibility.

290 Generally, internal transcribed spacers have been used for molecular identification of

291 fungal, but unfortunately, these sequences have little discrimination between $C$.

292 neoformans/gattii and their closely related species, impairing their use in species

293 differentiation by PCR methods[27]. To avoid non-specific amplification, we chose

294 CAP10 gene to design LAMP primers, mainly considering that it encoded a specific

295 capsular protein of $C$. neoformans/gattii, with little homology to their related species. The

296 results showed the specificity was $100 \%$, demonstrating the high specificity of the LAMP 297 systems that we established. 
The sensitivity of the LAMP assay for Cryptococcus cells is $100 \mathrm{CFU} / \mathrm{ml}$. Significantly,

299 it is of great importance for persons presenting early in the CM process with lower

300 burden of infection, because India ink's sensitivity was only $42 \%$ when the Cryptococcus

$301 \mathrm{CFU}$ value is $<1,000$ per $\mathrm{ml}$ of CSF[13]. Although the detection limit of LFA also

302 reached $100 \mathrm{CFU} / \mathrm{ml}$, LAMP assay could confirm cases which LFA had questionable

303 results. Moreover, the sensitivity of the LAMP assay for Cryptococcus genomic DNA

304 was $100 \mathrm{fg} / \mu \mathrm{l}$, which was little lower than those of Min Chen et al. (20 fg genomic DNA

305 tested by LAMP assay with turbidity method)[28] and Sara Gago et al. (2 fg genomic

306 DNA tested by Real-time PCR)[18,19]. The potential reason may be that we added the

307 cryptococcal DNA to the sterile CSF for further DNA extraction, which consumed a

308 portion of the DNA, while they used DNA to detect directly. Accordingly, we determined

309 the extraction efficiency of approximately 53.5\%. Therefore, the detection limit of

310 cryptococcal DNA in our study was similar to that of the Min Chen et al. study[28].

311 In clinical specimen verification, the total positive rate of $83 \mathrm{CSF}$ samples detected by

312 LAMP PCR was 91.6\% (76/83), which was higher than previously reported LAMP assay

313 with turbidity method $(87.1 \%, 74 / 85)[28]$ or real-time PCR $(90.7 \%, 39 / 43)$ results[19].

314 Although the positive rate of LFA titer in the range of 1:80-1:2,040 was $100 \%$, those of

315 lower titer $(1: 1-1: 40)$ was $33.3-90.0 \%$. The potential reason might be that dead

316 Cryptococcus cells continue to release capsular polysaccharide antigen, and the body

317 clears the antigen relatively slowly. Even after several months of effective treatment, the

318 results of LFA could still be positive. Therefore, the results of a patient with low LFA

319 titer could not truly reflect the burden of Cryptococcus in vivo.

320 In all, we established a rapid, accurate, sensitive, specific, and reproducible LAMP

321 assay to detect $C$. neoformans/gattii in CSF samples. This assay might be an alternative

322 method for rapid diagnosis of cryptococcal meningitis, especially for those with low 
323 cryptococcal load. Moreover, our research confirmed the feasibility of this LAMP system,

324 which made a good foundation for the further development of microfluidic chip.

325 Developing a microfluidic chip for real-life application of CM diagnosis remains a

326 challenge. Major limiting factors are low-concentration pathogen, complex sample

327 matrices and difficulties in extraction of Cryptococcus DNA on the chip. For removing

328 interfering substances and enrichment of pathogens, commonly used pretreatment tools

329 are centrifugation, filtration membranes or immune-based techniques [29]. However,

330 coupling the centrifugal device to the microfluidic chip is relatively complicated, and the

331 activity of immune-based methods is unstable. In contrast, the filter membrane has a

332 simple structure and can quickly and efficiently enrich pathogens at a level of $\sim 10^{-1} \mu \mathrm{m}$

333 (Cryptococcus was nearly $5 \mu \mathrm{m}$ in diameter)[30]. Benefit from these features, the filter

334 membrane structure was conveniently integrated into our chip, thus simplifying the entire

335 capture process. The core area of the microfluidic chip was composed of 4 duplicate

336 filtration membrane structures, aiming to expand the filtration area, improve fluid

337 throughput and prevent enrichment oversaturation caused by a single membrane structure.

338 Supporting pillars were used to support the filter membrane to improve filtration

339 efficiency. Pathogens in CSF samples were enriched through filtration membrane,

340 washed and purified, thus improving the system sensitivity and avoiding the

341 inconsistency of results due to the heterogeneity of sample matrix. In addition, the

342 successful extraction of on-chip cryptococcal nucleic acid is another key issue of this

343 microfluidic chip. Traditional methods often adopted beads mechanical capture method

344 or enzymatic cell wall lysis method. However, limited by the characteristics of the chip,

345 the mechanical method could not be applied. Accordingly, various experiments were

346 carried out to optimize on-chip Cryptococcus nucleic acid extraction. It was confirmed

347 that the use of standard Qiagen AL buffer and $0.5 \mathrm{M} \mathrm{NaOH}$ and incubated the lysing 
348 mixture at $65{ }^{\circ} \mathrm{C}$ for 5 minutes can improve the efficiency of nucleic acid extraction in

349 the chip. However, the above conditions were still insufficient. Because Cryptococcus is

350 different from other fungi, in addition to the common cell wall, its outer layer is

351 surrounded by a thick low electron density mucus capsule. Under the double protection of

352 the capsule and cell wall, Cryptococcusis much tougher and genomic DNA extraction is

353 more difficult. The removal of external structures to form protoplasts is essential for

354 nucleic acid extraction. Lyticase is a fungal cell wall lysis enzyme. We explored the

355 experimental effects of different concentrations of lyticase to optimize the best working

356 concentration. Moreover, protoplasts were very sensitive to external conditions and prone

357 to membrane rupture with changes in osmotic pressure. We used sorbitol buffer as the

358 osmotic pressure stabilizer to ensure a high protoplast formation rate. The results showed

359 that $40 \mathrm{U} / \mathrm{ml}$ lyticase digested Cryptococcus at $30{ }^{\circ} \mathrm{C}$ for 30 minutes in the $1 \mathrm{M}$ sorbitol

360 buffer environment, and the amplification effect was the best. The nucleic acid extract

361 was amplified isothermally for 45 minutes and the detection results could be successfully

362 read by the naked eye.

363 This microfluidic chip, integrating sample Cryptococcus enrichment, optimized nucleic

364 acid extraction and LAMP detection unit, streamlined reaction processes and reduced the

365 exposure risk of directly handling cryptococcal samples. It did not require any additional

366 instruments and provided a rapid, reliable, as well as high-efficiency approach. We

367 believed that this integrated chip truly realized the "sample-to-answer" application and

368 could be easily used for clinical cryptococcal prediagnosis.

\section{Acknowledgements}

371 We thank Professor Min Chen (Department of Dermatology, Shanghai Key Laboratory of

372 Molecular Medical Mycology, Shanghai Institute of Medical Mycology, Changzheng 
373 Hospital, Second Military Medical University, Shanghai, China) for giving us the

374 standard strain.

375

376 Disclosure statement

377 No potential conflict of interest was reported by the author(s).

378

379 Funding

380 This study was funded in part with the grants from the National Natural Science

381 Foundation of China (NSFC 81672105), the Program of Science and Technology

382 Commission of Shanghai Municipality (No.17JC1401000), the Shanghai Public Health

383 System Construction Three-Year Action Plan (2020-2022) Key Disciplines

384 (GWV-10.1-XK04), the Major Special Project of "Prevention and Control of Major

385 Infectious Diseases such as AIDS and Viral Hepatitis" (2018ZX10732401-003-016), the

386 Program of Science and Technology Commission of Shanghai Municipality

387 (19441903700), the National Natural Science Foundation of China (NSFC 21577019)

388 and the Shanghai Youth Clinical Medical Talent Training Program (Shanghai Medical

389 WeiJi 2016-04).

390

$391 \quad$ References

392 [1] May R, Stone N, Wiesner D, et al. Cryptococcus: from environmental saprophyte to

394 [2] Rajasingham R, Smith R, Park B, et al. Global burden of disease of HIV-associated 395 cryptococcal meningitis: an updated analysis. The Lancet Infectious diseases. $396 \quad 2017 ; 17(8): 873-881$. 
397 [3] Tsai W, Lien C, Lee J, et al. The prognostic factors of HIV-negative adult cryptococcal meningitis with a focus on cranial MRI-based neuroimaging findings. Journal of clinical neuroscience : official journal of the Neurosurgical Society of Australasia. 2018;55:57-61.

401 [4] Pyrgos V, Seitz A, Steiner C, et al. Epidemiology of cryptococcal meningitis in the US: 1997-2009. PloS one. 2013;8(2):e56269.

403 [5] Gago S, Serrano C, Alastruey-Izquierdo A, et al. Molecular identification, antifungal resistance and virulence of Cryptococcus neoformans and Cryptococcus deneoformans isolated in Seville, Spain. Mycoses. 2017;60(1):40-50.

[6] Li-Ping Zhu J-QW, Bin Xu, Xue-Ting Ou, Qiang-Qiang Zhang, Xin-Hua Weng. Cryptococcal meningitis in non-HIV-infected patients in a Chinese tertiary care hospital, 1997-2007. Med Mycol. 2010;48(4):570-579.

[7] Fang W, Fa Z, Liao W. Epidemiology of Cryptococcus and cryptococcosis in China. Fungal genetics and biology : FG \& B. 2015;78:7-15.

411 [8] Liu Y, Kang M, Wu S, et al. Different characteristics of cryptococcal meningitis between HIV-infected and HIV-uninfected patients in the Southwest of China. Medical mycology. 2017;55(3):255-261.

414 [9] Choi Y, Ngamskulrungroj P, Varma A, et al. Prevalence of the VNIc genotype of Cryptococcus neoformans in non-HIV-associated cryptococcosis in the Republic of Korea. FEMS yeast research. 2010;10(6):769-78.

417 [10] Mihara T, Izumikawa K, Kakeya H, et al. Multilocus sequence typing of 418 Cryptococcus neoformans in non-HIV associated cryptococcosis in Nagasaki, Japan. 
$419 \quad$ Medical mycology. 2013;51(3):252-60.

420 [11] Lahiri S, Manjunath N, Bhat M, et al. Clinical insights and epidemiology of central nervous system infection due to Cryptococcus neoformans/gattii species complexes: A prospective study from South India. Medical mycology. 2020;58(5):600-608.

CfD.

Cryptococcus

gattii

infection

statistics. https://wwwcdcgov/fungal/diseases/cryptococcosis-gattii/statisticshtml. 2020:CDC, Atlanta, GA.

[13] Rajasingham R, Wake R, Beyene T, et al. Cryptococcal Meningitis Diagnostics and Screening in the Era of Point-of-Care Laboratory Testing. Journal of clinical microbiology. 2019;57(1).

[14] Boulware D, Rolfes M, Rajasingham R, et al. Multisite validation of cryptococcal antigen lateral flow assay and quantification by laser thermal contrast. Emerging infectious diseases. 2014;20(1):45-53.

[15] M C, JR P. Cryptococcosis. Infectious disease clinics of North America. 2006;20(3):507-44, v-vi.

[16] Lindsley MD, Mekha N, Baggett HC, et al. Evaluation of a newly developed lateral flow immunoassay for the diagnosis of cryptococcosis. Clin Infect Dis. 2011 Aug;53(4):321-5.

437 [17] Rivera V, Gaviria M, Muñoz-Cadavid C, et al. Validation and clinical application of a molecular method for the identification of Cryptococcus neoformans/Cryptococcus gattii complex DNA in human clinical specimens. The Brazilian journal of infectious diseases : an official publication of the Brazilian 
442 [18] Tavares E, Azevedo C, Panagio L, et al. Accurate and sensitive real-time PCR assays using intergenic spacer 1 region to differentiate Cryptococcus gattii sensu lato and Cryptococcus neoformans sensu lato. Medical mycology. 2016;54(1):89-96.

[19] Gago S, Esteban C, Valero C, et al. A multiplex real-time PCR assay for identification of Pneumocystis jirovecii, Histoplasma capsulatum, and Cryptococcus neoformans/Cryptococcus gattii in samples from AIDS patients with

[20] X F, X F, B L, et al. Development of a singleplex PCR assay for rapid identification and differentiation of Cryptococcus neoformans var. grubii, Cryptococcus neoformans var. neoformans, Cryptococcus gattii, and hybrids. Journal of clinical microbiology. 2013;51(6):1920-3.

454 [21] Dou M, Sanjay S, Dominguez D, et al. Multiplexed instrument-free meningitis diagnosis on a polymer/paper hybrid microfluidic biochip. Biosensors \& bioelectronics. 2017;87:865-873.

[22] Dou M, Dominguez D, Li X, et al. A versatile PDMS/paper hybrid microfluidic platform for sensitive infectious disease diagnosis. Analytical chemistry. 2014;86(15):7978-86.

460 [23] Czilwik G, Messinger T, Strohmeier O, et al. Rapid and fully automated bacterial pathogen detection on a centrifugal-microfluidic LabDisk using highly sensitive nested PCR with integrated sample preparation. Lab Chip. 2015;15(18):3749-59. 
463 [24] Bridle H, Kersaudy-Kerhoas M, Miller B, et al. Detection of Cryptosporidium in miniaturised fluidic devices. Water Res. 2012 Apr 15;46(6):1641-61.

465 [25] Zhu Z, Zhang W, Leng X, et al. Highly sensitive and quantitative detection of rare pathogens through agarose droplet microfluidic emulsion PCR at the single-cell level. Lab Chip. 2012 Oct 21;12(20):3907-13.

[26] Swierczewska M, Liu G, Lee S, et al. High-sensitivity nanosensors for biomarker detection. Chem Soc Rev. 2012 Apr 7;41(7):2641-55.

[27] Espy M, Uhl J, Sloan L, et al. Real-time PCR in clinical microbiology: applications for routine laboratory testing. Clinical microbiology reviews. 2006;19(1):165-256.

[28] Chen M, Zhou J, Li J, et al. Evaluation of five conventional and molecular approaches for diagnosis of cryptococcal meningitis in non-HIV-infected patients. Mycoses. 2016;59(8):494-502.

[29] Strohmeier O, Keller M, Schwemmer F, et al. Centrifugal microfluidic platforms: advanced unit operations and applications. Chemical Society reviews. 2015;44(17):6187-229.

478 [30] Qi LX, Zhang; Yuhan, Yao; Wenwen, Jing; Sixiu, Liu; Guodong, Sui. . A novel microfluidic module for rapid detection of airborne and waterborne pathogens. Sensors \& Actuators B Chemical 2017:S092540051732244X. 
bioRxiv preprint doi: https://doi org/10.1101/2020 11.16.386045; this version posted November 17,2020 . The copyright holder for this preprint (which was not certified by peer review) is the author/funder, who has granted bioRxiv a license to display the preprint in perpetuity. It is made available under aCC-BY-NC-ND 4.0 International license.

\begin{tabular}{ll}
\hline Primer & Sequence Reference \\
\hline FIP & GTGGATTCCAGGCTGCTGATACATTTTCCGGCCCTTCCAAGTCTA \\
BIP & GATGGGGATGCTGAGTTGAGGAATTTTTCCGCCCAACAGTGAACA \\
F3 & AACGTCCACGCCTTCTTCT \\
B3 & GGTACTCACTCTCCATGTCG
\end{tabular}

Table 2 LAMP results for clinical samples from patients with proven CM

\begin{tabular}{|c|c|c|c|c|}
\hline Titres of $\mathrm{CrAg}$ & $\mathrm{N}$ & + & - & Positive rate $(\%)$ \\
\hline $1: 20480$ & 3 & 3 & 0 & $100 \%$ \\
\hline $1: 10240$ & 8 & 8 & 0 & $100 \%$ \\
\hline $1: 5120$ & 3 & 3 & 0 & $100 \%$ \\
\hline $1: 2560$ & 4 & 4 & 0 & $100 \%$ \\
\hline $1: 1280$ & 8 & 8 & 0 & $100 \%$ \\
\hline $1: 640$ & 8 & 8 & 0 & $100 \%$ \\
\hline $1: 320$ & 8 & 8 & 0 & $100 \%$ \\
\hline $1: 160$ & 10 & 10 & 0 & $100 \%$ \\
\hline $1: 80$ & 7 & 7 & 0 & $100 \%$ \\
\hline $1: 40$ & 10 & 9 & 1 & $90.0 \%$ \\
\hline $1: 20$ & 7 & 5 & 2 & $71.4 \%$ \\
\hline $1: 10$ & 3 & 1 & 2 & $33.3 \%$ \\
\hline $1: 5$ & 2 & 1 & 1 & $50.0 \%$ \\
\hline $1: 1$ & 2 & 1 & 1 & $50.0 \%$ \\
\hline Total & 83 & 76 & 7 & $91.6 \%$ \\
\hline
\end{tabular}



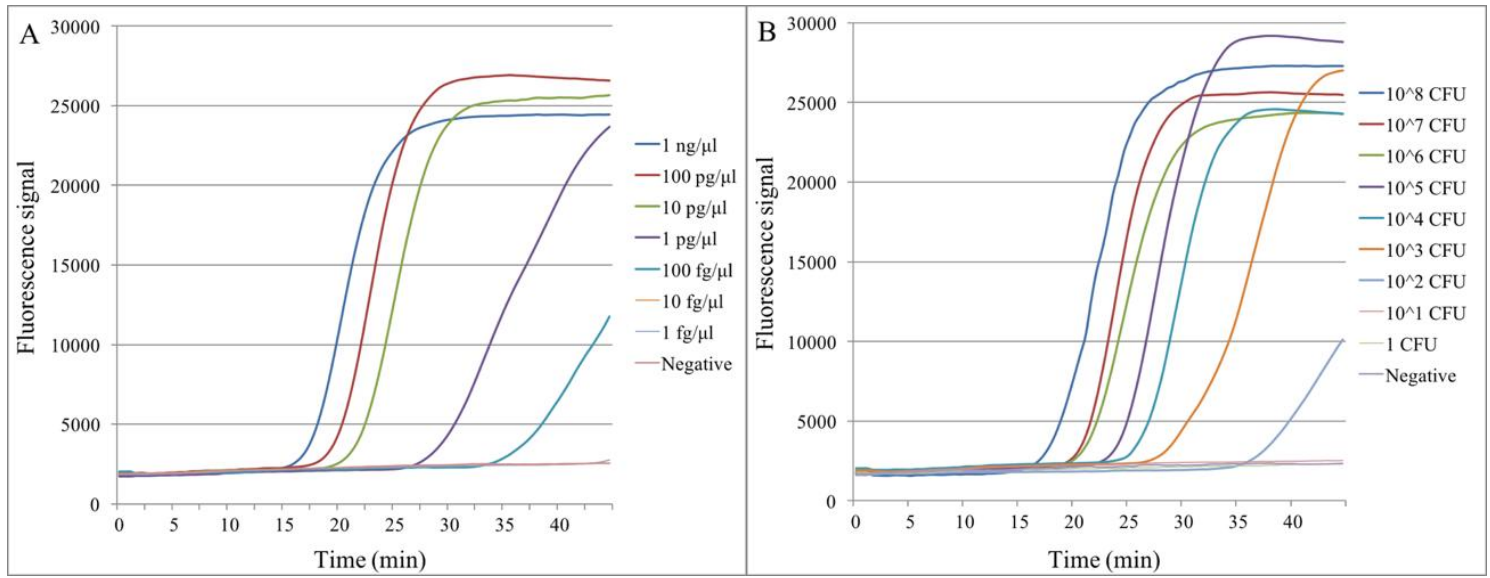

487

488 Figure 1. The detection limits of the LAMP assay.

489 A: Detection limit of Cryptococcus DNA. The blue, brown, green, purple, light blue, 490 yellow, light purple and pink lines represented 1ng/ $\mu 1,100 \mathrm{pg} / \mu \mathrm{l}, 10 \mathrm{pg} / \mu \mathrm{l}, 1 \mathrm{pg} / \mu \mathrm{l}$, $491100 \mathrm{fg} / \mu \mathrm{l}, 10 \mathrm{fg} / \mu \mathrm{l}, 1 \mathrm{fg} / \mu \mathrm{l}$ and negative templates, respectively. B: Detection limit of 492 Cryptococcus yeast suspension. The blue, brown, purple, light blue, yellow, light purple, 493 pink, light green and grey lines represented $10^{8}, 10^{7}, 10^{6}, 10^{5}, 10^{4}, 10^{3}, 10^{2}, 10,1 \mathrm{CFU}$ and 494 negative templates, respectively. 
bioRxiv preprint doi: https://doi org/10.1101/2020 11.16.386045; this version posted November 17, 2020. The copyright holder for this preprint (which was not certified by peer review) is the author/funder, who has granted bioRxiv a license to display the preprint in perpetuity. It is made available under aCC-BY-NC-ND 4.0 International license.

496

A

497

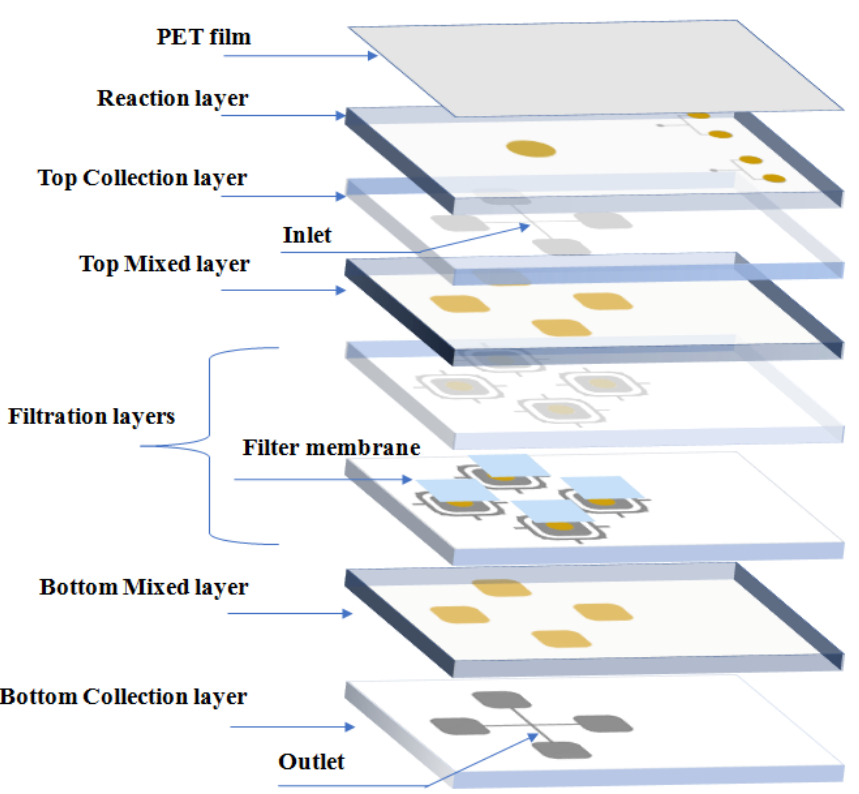

B

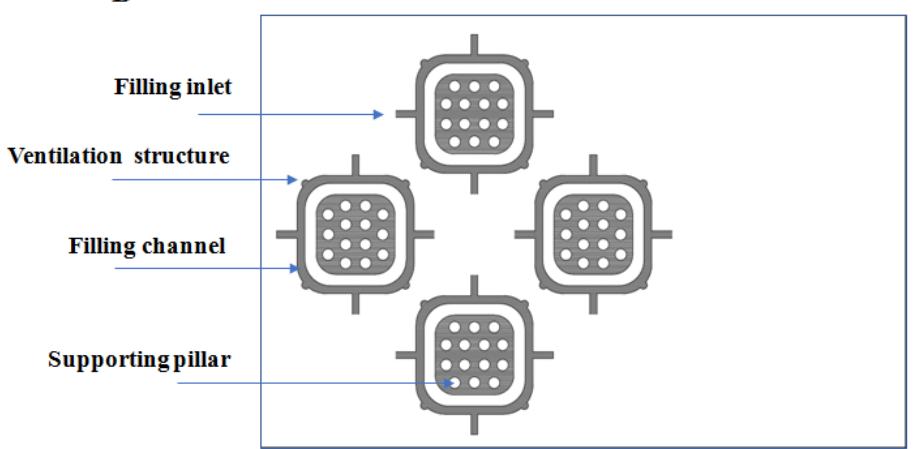

498

C

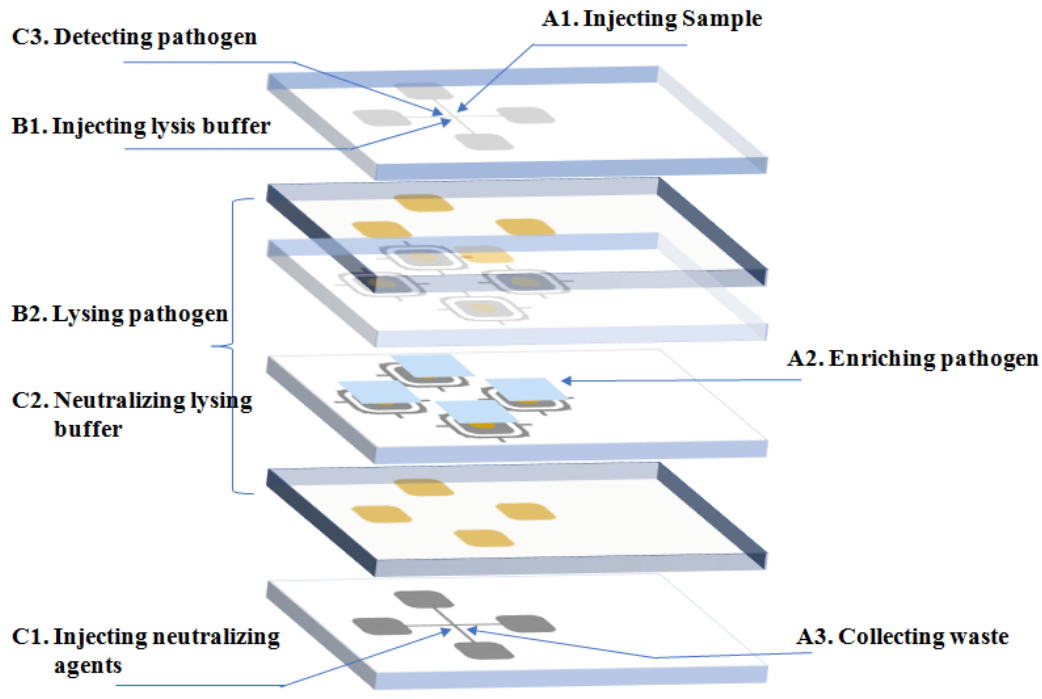

499 
500 Figure 2. Illustration of this microfluidic chip. A) The integrated chip has nine layers,

501 including functional layers and other different layers. The yellow part on these layers

502 indicates that the area has been punched through, and the gray part indicates that the area

503 is a fluid channel or a sinking part. B) This core area consisted of 4 duplicate parts.

504 PDMS/curing reagent mixtures were injected through the filling inlet and the protruding

505 small dots around the corner were used as a ventilation structure. Supporting pillars were

506 used to support the filter membrane to improve filtration efficiency. C) The nucleic acid

507 extraction process of the microfluidic chip included three steps. A. Pathogen enrichment

508 using filter membrane; B. Pathogen lysing with optimized lysis buffer; C. Pathogen

509 nucleic acid protection with neutralized agents.

510

511

512

A

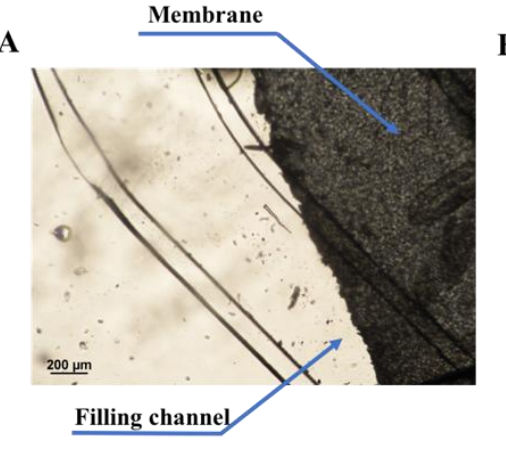

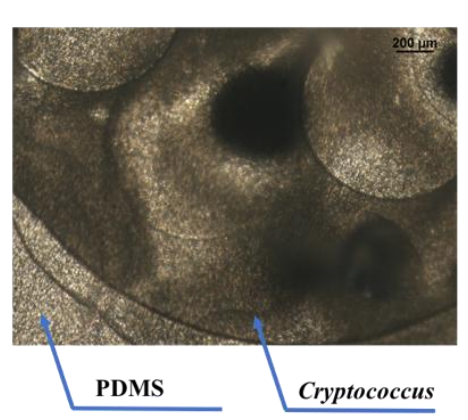

C

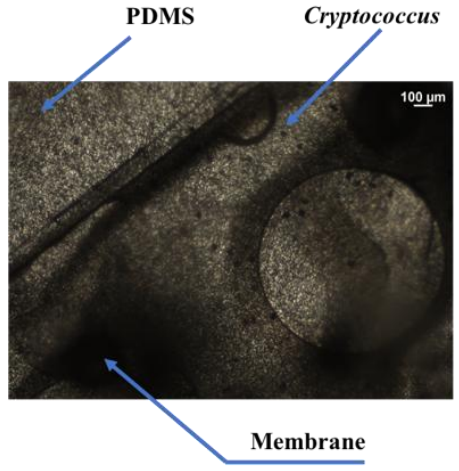

515 Figure 3. Filtration membrane enrichment images. A) Image of filtration membrane

516 structure before Cryptococcus injection; B) Image of filtration membrane structure after

517 Cryptococcus injection; C) Cryptococcus enriched by filtration membrane was stained by

518 black ink.

519 

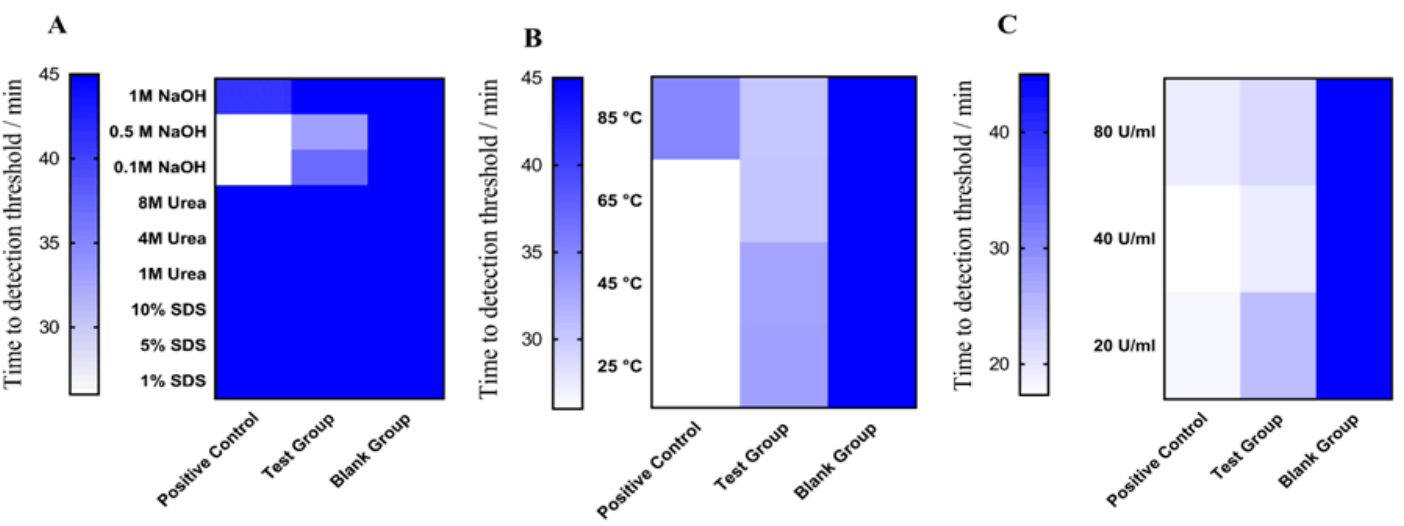

521 Figure 4. Nucleic acid fast extraction strategy. The positive control, test group and blank group, (referring to the nucleic acid extracted by the standard method, the sample and the DNase-free water respectively) were put into the lysis buffer, and then detected by the LAMP reaction after nucleic acid extraction. A) Three additives were tested for lysing capability and influence on LAMP reaction; B) Four temperature points were tested for lysing capability and influence on LAMP reaction. C) 1M Sorbitol buffer containing 

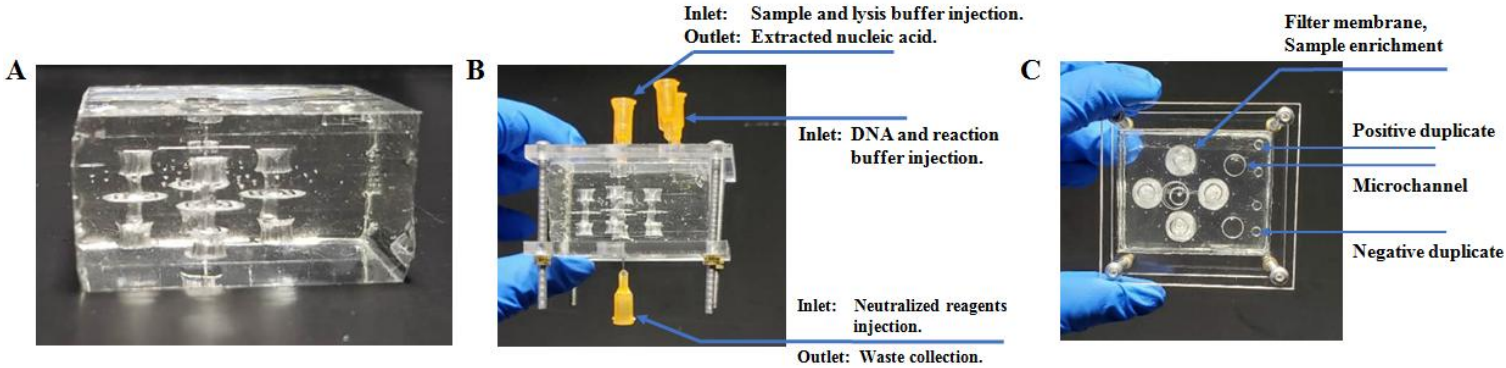

538 Figure 5. Picture of integrated chip. A) Integrated chip with PET film sealed; B)

539 Integrated chip inlet and outlet; C) Integrated chip top view, including filter membrane,

540 detection well, inlet, outlet and microchannels.

541

542

543

544

545

546

547

548

549

550

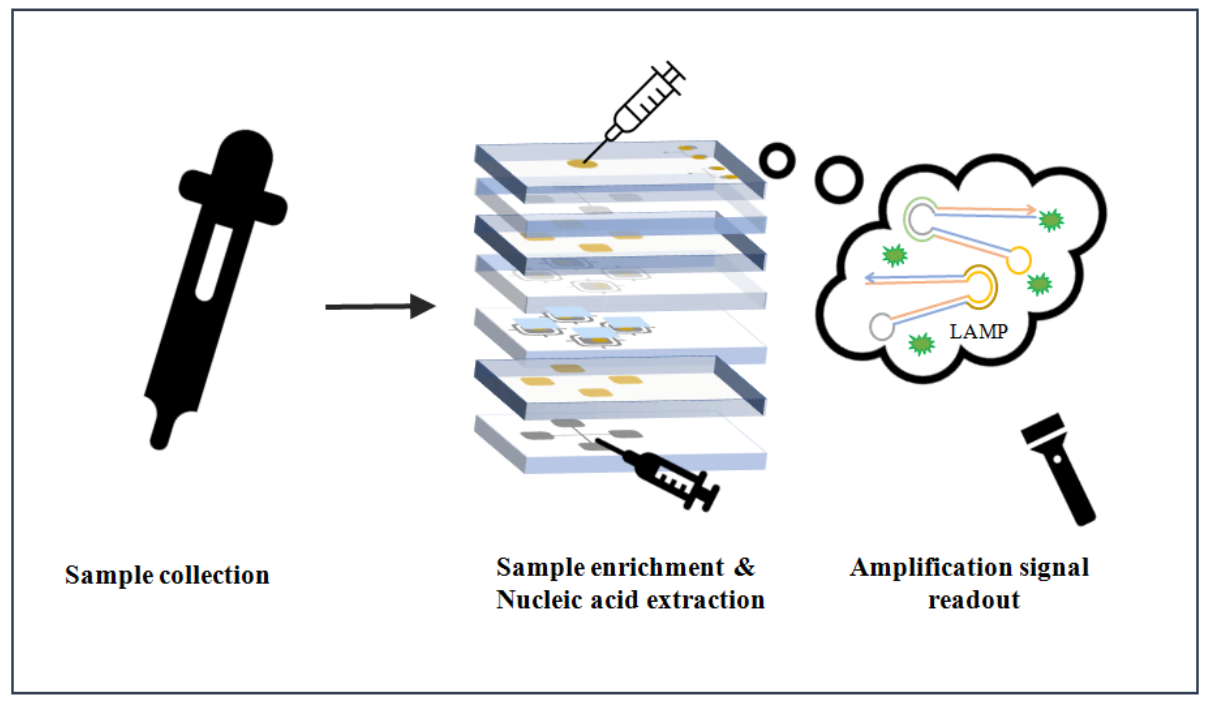

551 Figure 6. The whole process of detecting Cryptococcus on microfluidic chip. 
Table S1 LAMP results of CSF positive samples with other CNS infections

\begin{tabular}{|c|c|c|c|c|}
\hline & Samples & Culture results & $\mathbf{N}$ & LAMP results \\
\hline 1 & CSF & Acinetobacter baumannii & 2 & - \\
\hline 2 & CSF & Acinetobacter junii & 1 & - \\
\hline 3 & $\mathrm{CSF}$ & Klebsiella pneumoniae & 3 & - \\
\hline 4 & CSF & Staphylococcus aureus & 1 & - \\
\hline 5 & CSF & Staphylococcus hominis & 3 & - \\
\hline 6 & CSF & Staphylococcus capitis & 3 & - \\
\hline 7 & CSF & Staphylococcus epidermidis & 2 & - \\
\hline 8 & CSF & Staphylococcus haemolyticus & 1 & - \\
\hline 9 & CSF & Enterococcus faecalis & 3 & - \\
\hline 10 & CSF & Listeria monocytogenes & 2 & - \\
\hline 11 & CSF & Corynebacterium striatum & 1 & - \\
\hline 12 & CSF & Bacillus cereus & 1 & - \\
\hline 13 & CSF & Candida albicans & 3 & - \\
\hline 14 & CSF & Candida parapsilosis & 1 & - \\
\hline 15 & CSF & Candida orthopsilosis & 1 & - \\
\hline 16 & CSF & Candida glabrata & 2 & - \\
\hline 17 & CSF & Rhodotorula & 1 & - \\
\hline 18 & CSF & Nocardia pilaris & 1 & - \\
\hline 19 & CSF & Nocardia asiatica & 1 & - \\
\hline 20 & CSF & Mycobacterium tuberculosis & 4 & - \\
\hline 21 & CSF & Aspergillus flavus & 1 & - \\
\hline
\end{tabular}


22 CSF Candida parapsilosis.

Aspergillus niger

\begin{tabular}{lllll}
23 & CSF & Cysticercus & 1 & - \\
24 & Sterile CSF & Sterile CSF & 1 & - \\
25 & Ultrapure water & Ultrapure water & 1 & - \\
Totle & & & 42 & \\
\hline
\end{tabular}

Table S2 Standard $C$. neoformans/gattii strains

\begin{tabular}{llllll}
\hline & Standard strains & Serotype & Genotype & AFLP Type & LAMP \\
\hline 1 & C. neoformans var grubii & A & VNI & 1 & + \\
2 & C. neoformans var grubii & A & VNII & $1 \mathrm{~A}$ & + \\
3 & C. neoformans var neoformans & AD & VNIII & $1 \mathrm{~B}$ & + \\
4 & C. neoformans var neoformans & D & VNIV & 2 & + \\
\hline 5 & C. neoformans var gattii & B & VGI & 4 & + \\
6 & C. neoformans var gattii & B & VGII & 6 & + \\
7 & C. neoformans var gattii & B & VGIII & 5 & + \\
\hline 8 & C. neoformans var gattii & C & VGIV & 7 & + \\
\hline 9 & C. neoformans var grubii & A & VNI & 1 & + \\
\hline 10 & C. neoformans var neoformans & D & VNIV & 2 & + \\
\hline 11 & C. neoformans var grubii & A & VNI & 1 & + \\
\hline & & & & & + \\
\hline
\end{tabular}


Table S3 LAMP results of pure cultured isolates from different microorganisms

\begin{tabular}{|c|c|c|c|}
\hline & Isolates & $\mathbf{N}$ & LAMP results \\
\hline 1 & Acinetobacter baumannii & 3 & - \\
\hline 2 & Klebsiella pneumoniae & 5 & - \\
\hline 3 & Escherichia coli & 1 & - \\
\hline 4 & Staphylococcus epidermidis & 2 & - \\
\hline 5 & Staphylococcus hominis & 1 & - \\
\hline 6 & Staphylococcus haemolyticus & 1 & - \\
\hline 7 & Candida albicans & 2 & - \\
\hline 8 & Candida tropical & 2 & - \\
\hline 9 & Candida glabrata & 2 & - \\
\hline 10 & Candida krusei & 2 & - \\
\hline 11 & Candida guilliermondii; & 2 & - \\
\hline 12 & Candida parapsilosis & 2 & - \\
\hline 13 & Candida orthopsilosis & 2 & - \\
\hline 14 & Candida cornea & 1 & - \\
\hline 15 & Candida simmolone & 1 & - \\
\hline 16 & Rhodotorula & 2 & - \\
\hline 17 & Pichia norvegicus & 2 & - \\
\hline 18 & Issatchenkia orientalis & 1 & - \\
\hline 19 & Trichosporon asahii & 1 & - \\
\hline 20 & Asian nocardia & 2 & - \\
\hline 21 & Fovea nocardia & 1 & - \\
\hline 22 & Brazilian nocardia & 1 & - \\
\hline
\end{tabular}




\begin{tabular}{llll}
23 & Mycobacterium abscess & 1 & - \\
24 & European actinomycetes & 1 & - \\
25 & Penicillium chrysogenum & 1 & - \\
26 & Aspergillus fumigatus & 1 & - \\
27 & Aspergillus terreus & 1 & - \\
28 & Aspergillus niger & 1 & - \\
29 & Aspergillus oryzae & 1 & - \\
30 & Cyanobacterium marneffei & 1 & - \\
31 & Stephanoascus ciferrii & 1 & - \\
32 & Exophiala dermatitidis & 2 & - \\
Total & & 50 & \\
\hline
\end{tabular}

563 